\title{
Transformations of Feynman path integrals and generalized densities of Feynman pseudomeasures
}

DOI:

$10.1134 / \mathrm{S} 1064562416030169$

\section{Document Version}

Accepted author manuscript

Link to publication record in Manchester Research Explorer

\section{Citation for published version (APA):}

García-Naranjo, L. C., Montaldi, J., \& Smolyanov, O. G. (2016). Transformations of Feynman path integrals and generalized densities of Feynman pseudomeasures. Doklady Mathematics, 93, 282-285.

https://doi.org/10.1134/S1064562416030169

\section{Published in:}

Doklady Mathematics

\section{Citing this paper}

Please note that where the full-text provided on Manchester Research Explorer is the Author Accepted Manuscript or Proof version this may differ from the final Published version. If citing, it is advised that you check and use the publisher's definitive version.

\section{General rights}

Copyright and moral rights for the publications made accessible in the Research Explorer are retained by the authors and/or other copyright owners and it is a condition of accessing publications that users recognise and abide by the legal requirements associated with these rights.

\section{Takedown policy}

If you believe that this document breaches copyright please refer to the University of Manchester's Takedown Procedures [http://man.ac.uk/04Y6Bo] or contact uml.scholarlycommunications@manchester.ac.uk providing relevant details, so we can investigate your claim.

\section{OPEN ACCESS}




\title{
TRANSFORMATIONS OF FEYNMAN PATH INTEGRALS AND THE GENERALIZED DENSITIES OF FEYNMAN PSEUDOMEASURES
}

\author{
LUIS C. GARCÍA-NARANJO, JAMES MONTALDI, AND OLEG G. SMOLYANOV
}

\begin{abstract}
We consider the application of transformations of Feynman path integrals and Feynman pseudomeasures to problems of quantum anomalies. With our method we resolve a standing controversy in the literature.
\end{abstract}

Feynman pseudomeasures are defined to be linear functionals (distributions) on proper subspaces of functions defined on an infinite-dimensional vector space or manifold; the values that such functionals take on functions from their domains are called Feynman path integrals of those functions.

There exist two methods for obtaining formulae for transformations of Feynman pseudomeasures and, what is almost the same, for the Feynman path integrals. The first one of these methods can be traced back to a procedure developed for (only usual $\sigma$-additive) measures in [9] (see also an appendix in [4] written by V. Styablovskaya). This method uses the integration of the logarithmic derivatives of the distributions, along a family of transformations and subsequent integration of this derivative; some results in this direction were obtained in [2] and [7]. The second method uses the generalized densities of pseudomeasures; in a systematic form it was started in [1], but only for usual measures. In fact, this latter method gives a recipe to calculate the formulae while the former gives a way to prove the validity of the obtained formulae.

One of the possible applications of the obtained formulae for transformations of Feynman pseudomeasures is the explanation of the origin of the so-called quantum anomalies, i.e. symmetry breaking by the quantization procedure (cf. [5]). In particular one says that the quantum anomalies arise when after the quantization of a classical Lagrangian system, whose Lagrange function is invariant with respect to a collection of transformations one gets a quantum system which is not invariant anymore with respect to the same set of transformations. The explanation of the origin of the quantum anomalies, with full mathematical rigor, was first given in [6] . However, that paper mainly considers the logarithmic derivatives of the pseudomeasures and not the pseudomeasures themselves.

The aim of the present communication is twofold. On the one hand we extend the technique developed in [1] to pseudomeasures, and on the other, we apply this technique to discuss the origin of quantum anomalies in an alternative fashion to the approach taken in [6]. As it has already mentioned the first of the previously described methods has been

Key words and phrases. Feynman path integrals, transformation of Feynman pseudomeasures, quantum anomalies. 
extended to the Feynman type pseudomeasures in [2] and [7] (but without applications to quantum anomalies).

Our paper is organized as follows. After some preliminaries we investigate differential properties of pseudomeasures and introduce the notion of the generalized density of a pseudomeasure. Then we consider the application of the obtained results to the problem of quantum anomalies.

In the books [3] and [5] written by leading experts in mathematical physics, the authors suggest explanations of quantum anomalies which contradict each other. In [6] the contradiction has been explained using differential properties of the Feynman pseudomeasures; in what follows we consider the finite transformations of the same pseudomeasure.

Below we discuss mainly the algebraic structures of the considered problems and omit assumptions of analytical character.

\section{Preliminaries}

In his famous paper from 1948, Feynman represents a solution of the simplest Schrödinger equation by a limit of integrals over Cartesian powers of the spatial domain of the solution; after that, he interprets this limit as an integral over an infinite dimensional space of functions taking values in the same spatial domains. Finally, he notices that the integrand can be taken as the product of the exponent of the classical action and the initial data. In this paper by Feynman the action is presented in Lagrangian form, but in his next paper (1951) the action is presented in Hamiltonian form. Because of that it was necessary to consider, instead of the limit of integrals over cartesian powers of the spatial domains of the solution, the limit of the integrals over Cartesian powers of the cotangent bundles of the domain. But integration over infinite-dimensional spaces of functions, with values in proper subspaces, was understood by Feynman (without any mathematical definition) with respect to some analogues of Lebesgue measure, which do not exist in the infinite dimensional case (due to a theorem by A. Weil).

In order to embed this construction in a mathematical frame, one usually represents the exponent of the action as a product of two exponents: one of them defines a Feynman pseudomeasure (in our terminology it is the generalized density of the Feynman pseudomeasure) and the second one is a function whose product with the initial data is the integrand in the Feynman path integral (which is an integral, in some generalized sense, with respect to the the Feynman pseudomeasure).

Alternatively, one can (actually following the original ideas of Feynman) interpret the nonexisting analogue of Lebesgue measure as a pseudomeasure (in the style of the theory of distributions), using the original Feynman integrand: the initial data times the exponent of the classical action. 


\section{Differential properties of PSEudomeasures}

Let $E$ be a real locally convex space (LCS). A mapping from $E$ into another LCS is smooth along a Hilbert subspace ${ }^{1} H$ of $E$ if it is infinitely many times Hadamard differentiable along $H$. We assume that the following spaces of continuous linear mappings are equipped with the topology of compact convergence.

Let $\mathcal{D}$ be a complex LCS of smooth complex-valued functions on $E$ and let $\mathcal{D}^{\prime}$ be the space of all continuous linear functionals on $E$ equipped with the topology respecting the duality between $\mathcal{D}^{\prime}$ and $\mathcal{D}$ (in spite of such notation, we do not assume that if $E=\mathbb{R}^{n}$ then $\mathcal{D}$ is the space of smooth functions with compact supports). Elements of $\mathcal{D}^{\prime}$ are called pseudomeasures on $E$.

Let $\varepsilon>0$ and let $S$ be a mapping from $(-\varepsilon, \varepsilon)$ into a set of some Hadamard differentiable maps of $E$ into $E$ such that for every $\varphi \in \mathcal{D}$ and every $t \in(-\varepsilon, \varepsilon)$ the function

$$
(S(t))^{*} \varphi: E \ni x \mapsto \varphi(S(t) x)
$$

(the inverse image of $\varphi$ ) is an element of $\mathcal{D}$.

A pseudomeasure $\nu \in \mathcal{D}^{\prime}$ is said to be differentiable along $S$ if the function

$$
f_{S}:(-\varepsilon, \varepsilon) \ni t \mapsto(S(t))_{*} \nu
$$

is differentiable at $t=0$. Here $(S(t))_{*} \nu$ denotes the direct image of $\nu$ defined by

$$
(S(t))_{*} \nu(\varphi)=\nu\left((S(t))^{*} \varphi\right) .
$$

We call $f_{S}^{\prime}(0)$ the derivative of $\nu$ along $S$ and we denote it by $\nu_{S}^{\prime}$. If, moreover, there exists a function $\beta_{S}^{\nu}$ on $E$ such that $f_{S}^{\prime}(0)=\beta_{S}^{\nu}(\cdot) f(0)$ then $\beta_{S}^{\nu}$ is called the logarithmic derivative of the pseudomeasure $\nu$ along $S$.

A vector field $k$ on $E$ is a mapping of $E$ into $E$; if $k$ is a vector field on $E$ and $S$ is defined by

$$
S(t)(x)=x-t k(x)
$$

then the pseudomeasure $\nu$ on $E$ is said to be differentiable along the vector field $k$ if it is differentiable along $S$. The derivative (respectively the logarithmic derivative) of $\nu$ along $S$ is called the derivative (respectively the logarithmic derivative) of $\nu$ along the vector field $k$ and is denoted by $\nu^{\prime} k$ (respectively by $\beta_{k}^{\nu}$ ); so $\beta_{k}^{\nu}=\beta_{S}^{\nu}$ and $\nu^{\prime} k=\nu_{S}^{\prime}$.

If $k(x)=k_{0} \in E$ for all $x$ then the derivative of a pseudomeasure $\nu$ along $k$ is called the derivative along the vector $k_{0}$ and so on; in this case instead of the symbol $\beta_{k}^{\nu}$ we use the symbol $\beta^{\nu}(k, \cdot)$.

If $H$ is a Hilbert subspace of $E$ and if a pseudomeasure $\nu$ has a derivative along each $k \in H$, then the mapping $H \ni k \mapsto \nu^{\prime} k$ is linear (see [6] where the usual measures were considered). The corresponding vector valued distribution

$$
\mathcal{D} \ni \varphi \mapsto\left[k \mapsto\left(\nu^{\prime} k\right)(\varphi)\right]
$$

is called the derivative of $\nu$ along $H$.

\footnotetext{
${ }^{1}$ The definition of a Hilbert subspace of a LCS can be found in $[6]$
} 
If for any $k \in H$ there exists the logarithmic derivative of $\nu$ along $k$ then the mapping

$$
H \ni k \mapsto \beta^{\nu}(k, \cdot)
$$

is also linear. We denote it by $\beta^{\nu}$ and call it the logarithmic derivative along the subspace $H$. We stress that if $k$ is a vector field on $E$ for which $k(x) \in H$ for all $x$, then, in general $\beta_{k}^{\nu}(x) \neq \beta^{\nu}(k(x), x)$ (see [6] and the following theorem).

Theorem 2.1. Let $\nu$ be a pseudomeasure on $E$ which has the logarithmic derivative along a Hilbert subspace $H$ of $E$ and let $k$ be a vector field on $E$ taking values in $H$. Then, for the logarithmic derivative of $\nu$ along the vector field $k$ the following identity is valid:

$$
\beta_{k}^{\nu}(x)=\beta_{\nu}(k(x), x)+\operatorname{tr}\left(k^{\prime}(x)\right) .
$$

Proof. We present the idea of the proof. By the definition of the derivative of the $H$-valued distribution $k \cdot \nu$ one has, for any $\varphi \in \mathcal{D}$, the identity

$$
\left((k \cdot \nu)^{\prime}, \varphi\right)=-\left(k \cdot \nu, \varphi^{\prime}\right)
$$

where $(k \cdot \nu)^{\prime}$ and $\varphi^{\prime}$ are derivatives along $H$; so both sides of the identity are operators in $H$. Taking traces of those operators after applying the Leibniz rule to the left hand side one gets the following identity (we use some natural notation):

$$
\left(\beta^{\nu}(k(\cdot), \cdot) \nu, \varphi\right)+(\operatorname{tr}(k) \nu, \varphi)=-\left(\nu, \varphi^{\prime} k\right) .
$$

On the other hand, by direct calculations one can check that if $\nu$ is differentiable along the vector field $k$, then

$$
\left(\nu^{\prime} k, \varphi\right)=-\nu\left(\varphi^{\prime} k\right) .
$$

The theorem follows from the last two identities.

Corollary 2.2. If $\nu$ is a pseudomeasure and if $\beta^{\nu}(h, \cdot)=0$ for any $h \in H$ then

$$
\beta_{h}^{\nu}(x)=\operatorname{tr}\left(h^{\prime}(x)\right) \text {. }
$$

Such a pseudomeasure can be called a Lebesgue pseudomeasure.

\section{Generalized Densities of PSEudomeasures And AnOmalies}

We keep the assumptions and notation of the preceding section.

Definition 3.1. Let $\nu$ be a pseudomeasure which is differentiable along any direction in the Hilbert subspace $H$ of $E$. A generalized density of $\nu$ is a function $F_{\nu}: H \rightarrow \mathbb{R}$ whose logarithmic derivative along each $h \in H$ coincides with $\beta^{\nu}(h, \cdot)$.

If the logarithmic derivative of the pseudomeasure $\nu$ allows one to reconstruct $\nu$ then the generalized density also allows one to reconstruct $\nu$ (in this case $H$ is dense in $E$ ).

Now we describe the recipe to define the density of a pseudomeasure subjected to the transformation induced by a transformation $G$ of $E$.

Suppose there exists a function $S$ whose values are transformations such that $S(0)=I d$ and $S(1)=G$ (we also need to assume some "good enough" analytical properties of $G$ ). We assume that the values of the vector field generated by $S$ belong to $H$. Then, the formula from Theorem 2.1 is valid (see e.g. [9]). Using a calculation that is analogous 
to the ones performed in [9] (where only usual measures were considered) one obtains the following theorem.

Theorem 3.2. Let $\nu$ be a pseudomeasure on $E$ having logarithmic derivative along each $h \in H$, where $H$ is a dense Hilbert subspace of $E$ and assume that the preceding assumptions are valid. Then $G_{*} \nu=\Psi \nu$ where the function $\Psi$ (which can be called the density of $G_{*} \nu$ with respect to $\nu$ ) is defined to be the extension by continuity of the function $\Psi$ defined on its natural domain by

$$
\Psi(x)=\frac{F_{\nu}(G(x))}{F_{\nu}(x)} \operatorname{det}\left(G^{\prime}(x)\right)
$$

(see [1]).

Corollary 3.3. If $F_{\nu}$ is invariant with respect to $G$ (i.e. $\left.F_{\nu}(G(x))=F_{\nu}(x)\right)$ for each $x$ but $\operatorname{det}\left(G^{\prime}(x)\right)>1$ (or $<1$ ) for all $x$ then $G_{*} \nu \neq \nu$.

Corollary 3.4. From the discussion above, the Feynman path integral can be considered as the value that the "Lebesgue type pseudomeasure" (one whose logarithmic derivatives along any direction are equal to zero) takes on the function which is the product, of the exponent of the classical action, and of the initial data. If the action and the initial data are invariant with respect to a transformation $G$ then the Feynman path integral is invariant if $\operatorname{det}\left(G^{\prime}(x)\right)=1$ (see Theorem 3.6 below).

Corollary 3.5. Let $E_{t}, t>0$ be the space of all continuous real valued functions on $[0, t]$ vanishing at 0 , let $H_{t}$ be the Hilbert subspace of $E_{t}$ defined as the set of all absolutely continuous functions from $E_{t}$, with square integrable derivatives, equipped with the scalar product $(\cdot, \cdot)$ defined by $(f, g)=\int_{0}^{t} f^{\prime}(\tau) g^{\prime}(\tau) d \tau$ and let $\nu$ be the "classical" Feynman pseudomeasure on $E_{t}$ whose generalized density $F_{\nu}$ is defined by $F_{\nu}(\xi)=e^{\frac{i}{2} \int_{0}^{t}\left((\xi(\tau))^{\prime}\right)^{2} d \tau}$ where $\xi \in H_{t}$ (we do not use the subscript $t$ both in the notation $\nu$ and in some related notations below). Then the solution $\psi$ of the simplest Schrödinger equation, with a potential $V$ and an initial data $\psi_{0}$, is defined by $\psi(t, q)=\int_{E_{t}} \mathcal{F}^{q}(\xi) \nu(d \xi)$ where $\mathcal{F}^{q}(\xi)=e^{i \int_{0}^{t} V(\xi(\tau)+q) d \tau} \psi_{0}(\xi(t)+q)$.

The first statement of Corollary 3.4 implies the following identity $\int_{E_{t}} \mathcal{F}^{q}(\xi) \nu(d \xi)=$ $\int_{E_{t}} \mathcal{F}^{q}(\xi) F_{\nu}(\xi) \nu_{L}(d \xi)$ where $\nu_{L}$ - is the Lebesgue type pseudomeasure from Corollary 3.4 and the last integral, which can be called the Feynman path integral in Feynman's form, is the value that $\nu_{L}$ takes on the function $\mathcal{F}^{q}(\cdot) F_{\nu}(\cdot)$.

Corollary 3.6. The following identities hold:

$$
\text { (1) } \int_{E_{t}} \mathcal{F}^{q}(\xi) \nu(d \xi)=\int_{E_{t}} \mathcal{F}^{q}(G(\xi)) \frac{F_{\nu}(G(x))}{F_{\nu}(x)} \operatorname{det}\left(G^{\prime}(x)\right) \nu(d \xi),
$$

which is the change variable formula for the Feynman path integral;

$$
\text { (2) } \int_{E_{t}} \mathcal{F}^{q}(\xi) F_{\nu}(\xi) \nu_{L}(d \xi)=\int_{E_{t}} \mathcal{F}^{q}(G(\xi)) F_{\nu}(G(\xi)) \operatorname{det}\left(G^{\prime}(x)\right) \nu_{L}(d \xi),
$$

which is the change variable formula for the Feynman path integral in Feynman's form. 
Remark 3.7. The last statement of Corollary 3.3 actually coincides with the statement from [5] and contradicts what is said in [3]. In fact, using the Trotter-Chernoff Theorem (see [8] and references therein) one can obtain rigorous versions of the first statement from the 1948 paper by Feynman, about the representations of a solution of the Schrödinger equation, and of the similar statement from his 1951 paper. But the limits in the corresponding Feynman formulas coincide with the Feynman path integrals (in Feynman's form) of the exponent of the action times the initial data. From statements similar to the preceding corollary one finds that even if the action and the initial data are invariant with respect to a transformation $G$ but $\operatorname{det}\left(G^{\prime}(x)\right)>1$ (or $\left.<1\right)$ then the solution of the Schrödinger equation is not invariant with respect to the transformation. Moreover, any changing of the "measure of integration" referred to in [3] cannot make the solution invariant.

Remark 3.8. It would be interesting to investigate the notion of generalized density for pseudomeasures on infinite dimensional manifolds.

Acknowledgments. L.G.N was partially supported by a Royal Society Newton Advanced Fellowship held at the University of Manchester. The paper was written when O.G.S. visited the University of Manchester by the invitation of J.M. O.G.S. thanks the University of Manchester for hospitality and excellent work conditions. Also O.G.S. thanks the Russian foundation for basic research (grant 14-01-00516).

\section{REFERENCES}

[1] Montaldi J., Smolyanov O.G. , Transformations of measures via their generalized densities. Russian J. of Math. Physics, 2014, 21, No. 3, pp 1-10.

[2] Accardi, L., Smolyanov, O.G., Smolyanova, M.O. Change of variable formulas for infinite-dimensional distributions. (Russian) Mat. Zametki 60 (1996), no. 2, 288-292; translation in Math. Notes 60 (1996), no. 1-2, 212-215 (1997).

[3] Cartier P., DeWitt-Morette, C. Functional Integration, CUP, 2006.

[4] Daletskii, Yu.L., Fomin, S.V., Measures and differential equations in infinite-dimensional space. Translated from the Russian. With additional material by V.R. Steblovskaya, Yu.V. Bogdanskii and N.Yu. Goncharuk. Mathematics and its Applications (Soviet Series), 76. Kluwer Academic Publishers Group, Dordrecht, 1991.

[5] Fujikawa K., Suzuki, H., Path integrals and quantum anomalies. Oxford University Press, 2004, second printing 2013.

[6] Gough J., Ratiu T.S., Smolyanov O.G., Quantum anomalies and logarithmic derivatives of Feynman pseudo-measures. Doklady Math., 2015, 465, No. 6, (Russian version; the English version will also appear).

[7] Smolyanov, O.G., Trumen, A. Formulas for the change of variables for Feynman pseudomeasures. (Russian) Teoret. Mat. Fiz. 119 (1999), no. 3, 355-367; translation in Theoret. and Math. Phys. 119 (1999), no. 3, 677-686.

[8] Smolyanov, O.G., Tokarev, A.G., Truman, A. Hamiltonian Feynman path integrals via the Chernoff formula. J. Math. Phys. 43 (2002), no. 10, 5161-5171.

[9] Smolyanov, O.G., von Weizsäcker, H. Change of measures and their logarithmic derivatives under smooth transformations. C. R. Acad. Sci. Paris Sér. I Math., 1995, 321, No. 1, 103-108. 
Luis C. García-Naranjo: Departamento de Matemáticas y Mecánica, immas-Unam, Apdo Postal 20-726, Mexico City, 01000, Mexico

E-mail address: luis@mym.iimas.unam.mx

James Montaldi: School of Mathematics, University of Manchester, Manchester M13 9PL, UK

E-mail address: j.montaldi@manchester.ac.uk

Oleg G. Smolyanov: Lomonosov Moscow State University, Faculty of Mechanics and Mathematics, 119991 Moscow, Russia

E-mail address: smolyanov@yandex.ru 